\title{
Nanorobot Communication Techniques: A Comprehensive Tutorial
}

\author{
Adriano Cavalcanti \\ Tad Hogg \\ Bijan Shirinzadeh \\ Hwee C. Liaw \\ CAN Center for Automation in Nanobiotech \\ Sao Paulo, SP 01540, Brazil \\ email: adrianocavalcanti@canbiotechnems.com \\ HP Labs \\ Palo Alto, CA 94304, USA \\ email: tad_hogg@hp.com

\begin{abstract}
Robotics and Mechatronics Research Lab., Dept. of Mechanical Eng., Monash University
Clayton, Melbourne, VIC 3800, Australia

emails: \{bijan.shirinzadeh, hwee-choo.liaw\}@eng.monash.edu
\end{abstract}

\begin{abstract}
This work presents chemical communication techniques for nanorobots foraging in fluid environments relevant for medical applications. Unlike larger robots, viscous forces and rapid diffusion dominate their behaviors. Examples range from modified microorganisms to nanorobots using ongoing developments in molecular computation, sensors and motors. The nanorobots use an innovative methodology to achieve decentralized control for a distributed collective action in the combat of cancer. A communication approach is described in the context of recognizing a single tumor cell in a small venule as a target for medical treatment. Thus, a higher gradient of signal intensity of E-cadherin is used as chemical parameter identification in guiding nanorobots to identify malignant tissues. A nanorobot can effectively use chemical communication to improve intervention time to identify tumor cells.
\end{abstract}

Keywords - Biomedical engineering, cancer, control systems, E-cadherin signal, endothelial cell, nanomechatronics, nanomedicine, nanorobots, nanotechnology, transducers, virtual reality.

\section{INTRODUCTION}

$\mathbf{N}$ anorobots with sizes comparable to bacteria could provide many novel capabilities through their ability to sense and act in microscopic environments. Particularly interesting are biomedical engineering applications [1], [2], where nanorobots and nanoscale-structured materials inside the body provide significant improvements in diagnosis and treatment of disease [3], [4], [5]. The rapid progress in building nanoscale devices should enable a wide range of capabilities [6]. For example, ongoing development [7], [8], [9], of molecular-scale electronics, sensors and motors provides components to enable nanorobots [10]. Demonstrations of programmable bacteria [11] can produce computation capability for nanodevices. The ways to enable Nano-Build Hardware Integrated Systems has been demonstrated and manufacturing techniques are advancing gradually [9]. The development of nanosystems for control of nanorobots to perform specific tasks in medicine may also enable improvements for nanotechnology automation [12], [13]. The next level of challenge for nanotechnology development may address intelligent control system with device manufacturing and automation in a broad sense [14], [15].

We present a comprehensive work on techniques for nanorobots communication based on extensive numerical results and real time 3D simulation. The approach in this paper is applied to the following biomedical problem: the nanorobots with embedded chemical sensors perform the detection of a single tumor cell in a small venule [16], [17], [18]. The venule is one among many types of vessels from the human body. Integrated nanosensors can be utilized for such a task in order to find intensity of E-cadherin signals [19], [20]. Brownian motion has a direct influence in a microfluid workspace, which for active communication makes stigmergy the natural way for near distance interaction [21]. Thus, communication and nanorobot control reacting to changes in the environment is quite appropriate for our study as described in this paper.

\section{BEHAVIOR IN FLUID MICROENVIRONMENTS}

We consider nanorobots operating in small blood vessels. The fluid in the vessels contains numerous cells, several microns in diameter. Viscosity dominates the nanorobots motion through the fluid in the environment, with physical behaviors quite different from our experience with larger organisms and robots [22], [23]. The ratio of inertial to viscous forces for an object of size $R$ moving with velocity $v$ through a fluid with viscosity $\eta$ and density $\rho$ is given by the Reynolds number as follows: 


$$
\operatorname{Re} \equiv R \rho v / \eta
$$

Typical values for density and viscosity in blood plasma are represented by equations 2 and 3 respectively.

$$
\begin{gathered}
\rho=1 \mathrm{~g} / \mathrm{cm}^{3} \\
\eta=10^{-2} \mathrm{~g} / \mathrm{cm} . \mathrm{s}
\end{gathered}
$$

Flow speeds in small blood vessels are about $1 \mathrm{~mm} / \mathrm{s}$. This is also a reasonable speed for nanorobot motion with respect to the fluid [3], providing $\operatorname{Re} \approx 10^{-3}$ for a 1-micron nanorobot, and thus viscous forces dominate. Consequently, nanorobots applying a locomotive force quickly reach a desired velocity in the fluid. Hence, applied force is proportional to velocity rather than the direct correlation applied in the acceleration of Newton's law $F=m a$. Diffusion arising from thermal motion of molecules (Brownian motion) is also important. Depending on the object's size, the diffusion coefficient $D$ characterizes the resulting random motion. In a time $t$, the root-mean-square displacement due to diffusion may be defined as:

$$
k=\sqrt{6 D t} .
$$

For a nanorobot $\approx 1 \mu m$ operating at body temperature, this displacement is $\approx \sqrt{t}$ microns with $t$ measured in seconds. Brownian motion also randomly changes the nanorobot orientation. Chemicals have much larger diffusion coefficients than nanorobots. Because displacement grows as $\mathrm{O}(\sqrt{t})$ instead of linearly in $t$, diffusion is fast at short distances and relevant for coordinating activity among nearby nanorobots, but slow over long distances. Chemicals can signal medically relevant events [24], and also be used for nanorobots communication. Communication ideally involves chemicals not otherwise found in the body (to produce low signal noise level) which are biologically inert over the relevant time scale of the nanorobot task, and can later be cleared from the body by existing biological processes [3].

\section{NANOROBOT DESIGN}

Virtual Reality was considered a suitable approach for nanorobot design and for the use of macro- and micro-robotics concepts given certain theoretical and practical aspects that focus on its domain of application. The nanodevice design must be robust enough to operate in an aqueous environment with movement having six-degrees of freedom. The nanorobot design is derived from biological models and is comprised of components such as molecular sorting rotors and a robot arm (telescoping manipulator) [25]. The nanorobot exteriors considered is comprised of diamondoid new material [26], [27] to which may be attached an artificial glycocalyx surface that minimizes fibrinogen (and other blood protein) absorption and bioactivity, thus ensuring sufficient biocompatibility for

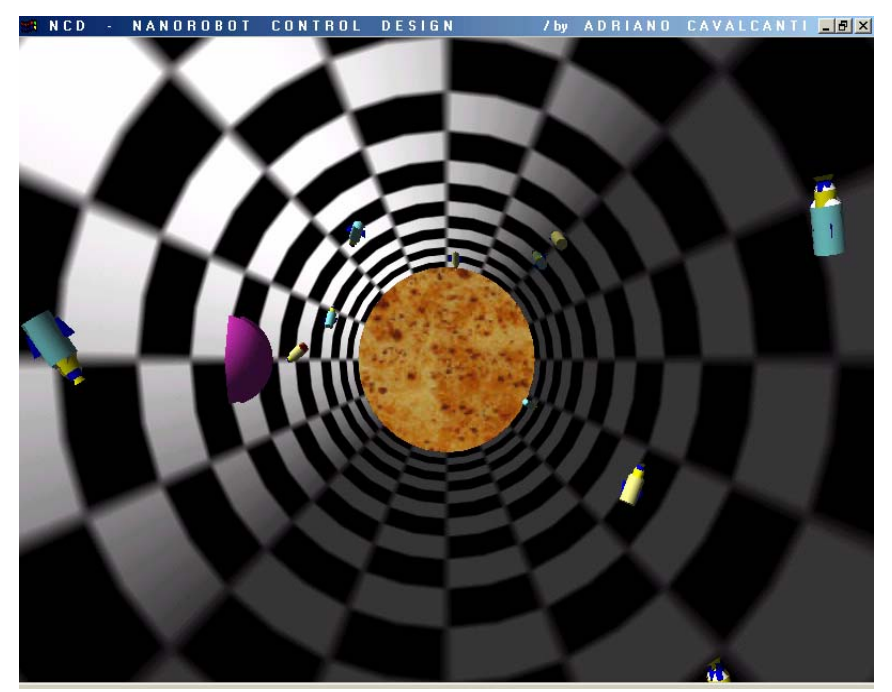

Figure 1: Vein internal view without the red cells. The tumor cell is the target represented by the pink sphere located left at the wall. All the nanorobots swim near the wall to detect cancer signals.

the nanorobot to avoid immune system attack [3]. Different molecule types are distinguished by a series of chemotactic sensors whose binding sites have a different affinity for each kind of molecule [3].

The control system must ensure a suitable performance. It can be demonstrated with a determined number of nanorobots responding as fast as possible for a specific task based scenario. In our work, we consider nanorobots flowing in a blood vessel with a small target area on the wall emitting a specific chemical. Manufacturing better sensors and actuators with nanoscale sizes is advancing [28], [9]. The nanorobots, designed with sensors for this chemical, must find the source in a vessel wall. In the 3D workspace the target has surface chemicals allowing the nanorobots to detect and recognize it.

\section{PHYSICAL PARAMETERS}

The microenvironments of the circulatory system vary considerably in size, flow rates, and other physical properties. Moreover, chemicals in the blood have a range of diffusion coefficients, and there is a range of plausible designs for the nanorobots.

We use typical values for these properties, but our control techniques can be modified for other values such as adjusting detection thresholds. Small vessels have diameters of up to several tens of microns, and lengths of about a millimeter. The workspace used in the simulator comprised an environment consisting of a segment of the vessel of length $L$ with a small target region on the wall emitting a chemical into the fluid (Fig. 1). Cells and nanorobots continually enter one end of the workspace along with the fluid flow. We treat any nanorobots not responding while within the workspace as if they did not detect any signal, so they flow with the fluid as it leaves the workspace. Thus, we choose the workspace length sufficient 
to include the region where the chemical from the target is significantly above the background level. The cells occupy about 1/5-th of the workspace volume, a typical hematocrit value for small blood vessels.

Table I lists the details included in the simulator for rendering in real time the $3 \mathrm{D}$ environment, including nanorobots and chemical signal parameters. We treat the nanorobots as cylinders, $1 \mu \mathrm{m}$ in length and $0.5 \mu \mathrm{m}$ in diameter. Most of the cells are red blood cells, with diameter $6 \mu \mathrm{m}$. The number densities of platelets and white blood cells are about 1/20-th and 1/1000-th that of the red cells, respectively. The nanorobot density equals $10^{12}$ nanorobots in the entire 5-liter blood volume of a typical adult. Thus a similar number of nanorobots may be used in medical applications [3]. The total mass of all the nanorobots is about $0.2 \mathrm{~g}$. Due to fluid drag and the characteristics of locomotion in viscous fluids, nanorobots moving through the fluid at $\approx 1 \mathrm{~mm} / \mathrm{s}$ dissipate a picowatt [29]. Thus, if all the nanorobots moved simultaneously they would use about one watt, compared to a typical person's 100-watt resting power consumption.

As a specific example, we consider a typical protein produced in response to injury, with concentration near the injured tissue of $\approx 30 \mathrm{ng} / \mathrm{ml}$ and background concentration in the bloodstream about 300 times smaller. A typical molecular weight of $10^{4}$ Dalton leads to the parameter values for the chemical signal in Table I. This choice provides an interesting nanorobot task, though we could equally well study tasks involving chemicals with different concentrations relevant for other biomedical engineering applications [21]. In our study, the chemical signal was taken to be produced uniformly over the target region at the rate $Q$. The background concentration, listed in Table I, is a significant sensory parameter, because the signal rapidly dilutes as it diffuses from the source.

\section{NANOROBOT BEHAVIORS CONTROL}

In our research, with aims of addressing analyses and validation for feasible nanorobot control design automation, the Nanorobot Control Design (NCD) simulator was developed, which is software for nanorobots in environments with fluids dominated by Brownian motion and viscous rather than inertial forces. We examine several practical control techniques for nanorobot motions. First, as a point of comparison, we use the nanorobots' small Brownian motions to find the target by random search. In a second method, the nanorobots monitor for chemical concentration significantly above the background level. After detecting the signal, a nanorobot estimates the concentration gradient and moves toward higher concentrations until it reaches the target. In the third approach, nanorobots at the target release another chemical which others use as an additional guiding signal to the target. With our signal concentrations, only nanorobots

\begin{tabular}{|c|c|}
\hline \multicolumn{2}{|c|}{$\begin{array}{c}\text { TABLE I } \\
\text { PARAMETERS }\end{array}$} \\
\hline \multicolumn{2}{|c|}{ Chemical signal } \\
\hline production rate & $Q=10^{4}$ molecule $/ \mathrm{s}$ \\
\hline diffusion coefficient & $D=100 \mu m^{2} / s$ \\
\hline background concentration & $6 \times 10^{-3}$ molecule $/(\mu \mathrm{m})^{3}$ \\
\hline Parameter & Nominal value \\
\hline average fluid velocity & $v=1000 \mu \mathrm{m} / \mathrm{s}$ \\
\hline vessel diameter & $d=20 \mu m$ \\
\hline workspace length & $L=50 \mu m$ \\
\hline density of cells & $2.5 \times 10^{-3} \mathrm{cell} /(\mu \mathrm{m})^{3}$ \\
\hline density of nanorobots & $2 \times 10^{-4} \mathrm{robot} /(\mu \mathrm{m})^{3}$ \\
\hline
\end{tabular}

passing within a few microns of the target are likely to detect the signal. Thus, we improve the response by having the nanorobots maintain positions near the vessel wall instead of floating throughout the volume flow in the vessel (Fig. 1). In the render modeling was used a vein wall with grid texture to enable better depth and distance perception in the 3D workspace. A key choice in chemical signaling is the measurement time and detection threshold at which the signal is considered to be received. Due to background concentration, some detection occurs even without the target signal. As a guide for the choice of threshold, we use the diffusive capture rate $\alpha$ for a sphere of radius $R$ in a region with concentration as:

$$
\alpha=4 \pi D R C
$$

where the concentration for other shapes such as cylinders are about the same [29]. With independent random motions for the molecules, detection over a time interval $\Delta t$ is a Poisson process with mean value $\alpha \Delta t$. Using Table I, $\alpha \approx 0.5$ molecule $/ s$ at the background concentration and $\approx 150$ near the source. With the target on the vessel wall, fluid velocity near the target is lower than the average velocity $v$ in Table I. When objects occupy only a small fraction of the volume the velocity at distance $r$ from the center of the vessel is:

$$
w=2 v\left(1-(r /(d / 2))^{2}\right),
$$

and with the cells, the velocity shows somewhat a parabolic flow [3], but similar enough for this parabolic profile to give a useful design guideline.

The control design has to avoid nanorobots to miss the target as well as to spend power in unnecessary active locomotion. Obviously, after detecting the signal, for the nanorobot to move far against the bloodstream, and go around numerous blood cells, to reach the target may waste precious time and energy. Thus, a reasonable design choice is for nanorobots to respond within at most $10 \mu \mathrm{m}$ downstream of the target. A 


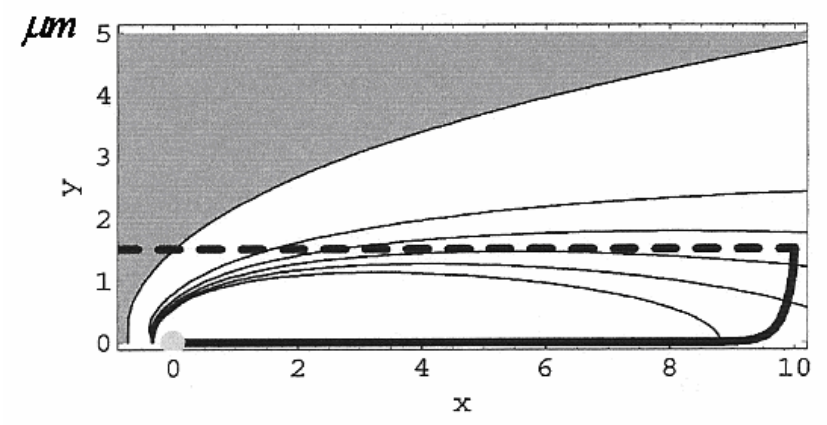

Figure 2: Nominal behavior of a nanorobot passing above the target (small gray circle) with the fluid moving to the right. Thick dashed line shows initial passive motion, lasting about $10 \mathrm{~ms}$, as the nanorobot determines signal concentration is significantly above background. Distances are in microns.

nanorobot $2 \mu \mathrm{m}$ from the wall encounters fluid velocity $\approx 400 \mu \mathrm{m} / \mathrm{s}$. Therefore, it takes about $30 \mathrm{~ms}$ to move $10 \mu \mathrm{m}$, during which time it will detect, on average, $\approx 3$ signal molecules while the background concentration has $\approx 1 \%$ probability to give even a single detection in this time. Thus, to save power with sensor processing, the activation threshold to detect signals is setup for intervals of $30 \mathrm{~ms}$. In the measurement to estimate the concentration gradient, the sensors are positioned on the surface of nanorobot's extremities. After detecting the signal, the nanorobot estimates the direction to the target from the concentration gradient. The process consists of alternate short movements with random changes in direction, at a rate depending on how the concentration changes during the move. If no signal was detected, the nanorobot just keeps flowing with the bloodstream saving power consumption.

In analogy with quorum sensing in bacteria, from monitoring the concentration of a signal from others, a nanorobot can estimate the number of nanorobots at the target. So, the nanorobot uses this information to determine when enough nanorobots are at the target, thereby terminating any additional "attractant" signal a nanorobot may be releasing. In our investigation, the nanorobots stop attracting others once enough nanorobots have responded. The amount is considered enough when the target region is densely covered by nanorobots. Thus, for investigation purposes, values of $N=\{10$, 20\} were set up in the simulator as a reasonable amount of nanorobots to the plaque target lesion. A feasible continuation of this procedure would be to have the nanorobots emit a different signal that others, not already at the target, interpret as an indication they no longer need to respond, thereby leaving them free to continue monitoring for other target areas. Detecting multiple signaling chemicals requires sensors for more than one chemical.

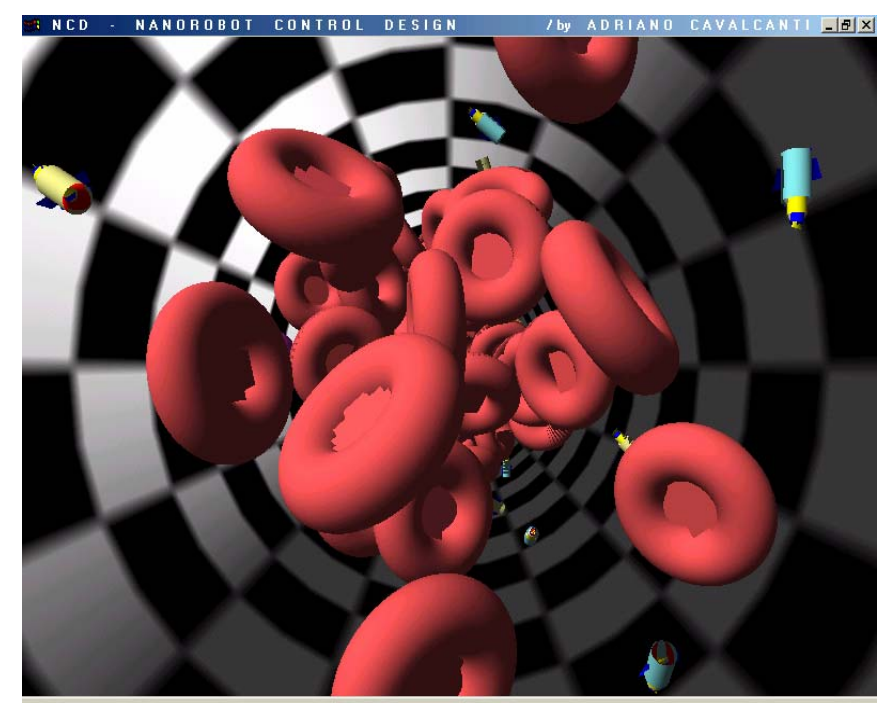

Figure 3: View of simulator workspace showing the vessel wall, cells and nanorobots. The nanorobot is considerably smaller than the $6 \mu \mathrm{m}$ cell diameter.

The following control methods were considered:

- Random: nanorobots moving passively with the fluid reaching the target only if they bump into it due to Brownian motion.

- Follow gradient: nanorobots monitor concentration intensity for E-cadherin signals, when detected, measure and follow the gradient until reaching the target. If the gradient estimate subsequent to signal detection finds no additional signal in $50 \mathrm{~ms}$, the nanorobot considers the signal to be a false positive and continues flowing with the fluid.

- Follow gradient with attractant: as above, but nanorobots arriving at the target, they release in addition a different chemical signal used by others to improve their ability to find the target.

The third technique involving communication among the nanorobots is quite suitable to improve the nanorobots' behavior performance. By comparing these techniques, we can evaluate the benefit of chemical communication among nanorobots to work on typical biomedical applications.

\section{SIMULATOR RESULTS}

To illustrate some design choices, we first examine an analytically solvable version of the fluid environment and then describe the results from the simulator. Consider a fluid moving uniformly with velocity $v$ in the positive $\mathrm{x}$-direction past a plane. It contains a point source of chemical produced at a rate $Q$, which is the chemical signal as molecules per second. The diffusion coefficient is represented by $D$, and the diffusion equation is:

$$
D \nabla^{2} C=v \partial C / \partial x
$$

with the boundary conditions of a steady point source at the 


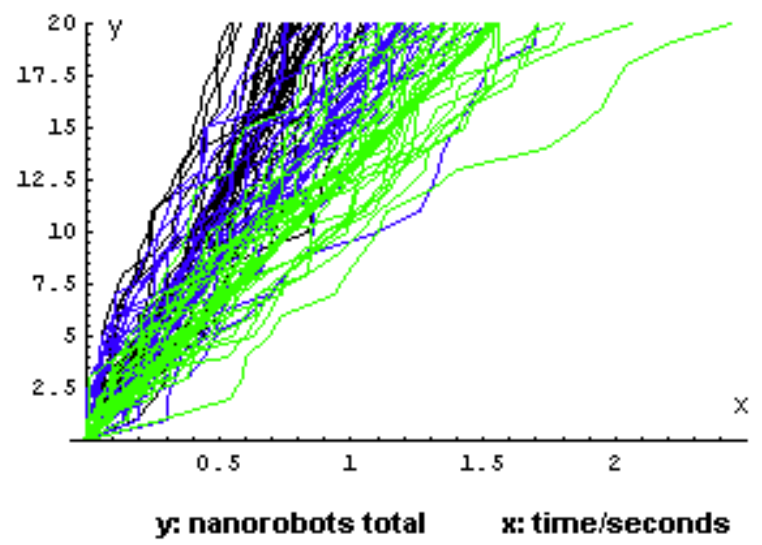

Figure 4: Detailed simulation depicting 90 experiments with the amount of $1 / 3$ for each control method. Respective colors represent the cases for nanorobot behavior based on (a) dark for gradient with attractant, (b) blue for follow gradient, or (c) green for random motion.

origin and no net flux across the boundary plane at $y=0$, determines the steady-state concentration $\mathrm{C}$, which is molecules per $\mu m^{3}$ or chemical concentration at point $(x, y, z)$ :

$$
C(x, y, z)=\frac{Q}{2 \pi D r} e^{-v(r-x) /(2 D)}
$$

where

$$
r=\sqrt{x^{2}+y^{2}+z^{2}}
$$

is the distance to the chemical signal source [29].

Fig. 2 is an illustration of nanorobot behavior. The fluid flow pushes the concentration of the diffusing signal downstream. Consequently a nanorobot passing more than a few microns from the source won't detect the signal while it is still relatively near the source. As an example, considering the parameters from Table I, when nanorobots passing close enough, they detect on average the higher signal concentration within about $10 \mathrm{~ms}$. Thus, keeping their motion near the vessel wall, the signal detection happens after these have moved at most $10 \mu \mathrm{m}$ past the source. Therefore, it provides about 5 nanorobot/s arriving at the tumor cell in the small venule.

Eq. (8) also illustrates a design trade-off for chemical signals the nanorobots could release. Instead of the diffusion coefficient associated with the chemical from the target, such additional signals would use other molecules which could, by design, have a different diffusion coefficient. From Eq. (8), the effect of the fluid motion becomes significant at distances beyond $O(D / v)$. Thus, notwithstanding the fluid flow, larger diffusion constants allow further spread upstream. On the other hand, the $O(1 / D)$ overall factor in Eq. (8) means lower concentrations. Furthermore, the concentration of the new signal is time dependent since the source strength increases as more nanorobots reach the target and the signal from each nanorobot requires time $O\left(r^{2} / D\right)$ to reach a distance $r$.
TABLE II

NANOROBOTS: TIME IN SECONDS TO REACH THE TARGET

\begin{tabular}{ccc}
\hline Control method & 10 robots & 20 robots \\
\hline Random motion & $0.73(0.18)$ & $1.47(0.28)$ \\
Follow gradient & $0.54(0.17)$ & $1.14(0.24)$ \\
Gradient with "attractant" & $0.46(0.13)$ & $0.79(0.14)$ \\
\hline
\end{tabular}

Therefore, faster diffusion results in lower concentrations, requiring more time for other nanorobots to determine gradients. Hence, chemical diffusion could be more efficient for nanorobot communication, if the signals are increasing in a steady, constant and progressively manner.

Nanorobots passing within $\approx 0.1 \mu \mathrm{m}$ of the target usually bump into it. Those passing within a few microns often detect the signal, which spreads a bit further upstream and away from the single tumor due to the slow fluid motion near the venule's wall and the cells motion. Nanorobots close to the wall also benefit from the slower fluid motion by having more time to detect the signal, as discussed previously. Thus, the present 3D simulation provides guidelines for nanorobot communication and activation control, as well as for sensor manufacturing design. We use an "attractant" signal with the same value of $D$ as the original signal. Each nanorobot can release at one-tenth the rate of the target over the times considered here.

Distinct performances were observed throughout a set of analyses obtained from the NCD software, where the nanorobots use also chemical sensors as the communication technique to interact dynamically with the $3 \mathrm{D}$ environment, and to achieve a more successful collective coordination. Fig. 3 shows the virtual environment in our study, comprised a small venule vessel which contains nanorobots, the red blood cells (RBCs) and a single tumor cell, which is the target area on the vessel wall. Here, the target area is overleaped by the RBCs. In the simulation, the nanorobots search for possible small cancer tumor into the workspace crowded by RBCs.

In Fig. 4 it could be observed in a detailed fashion the information about the nanorobots behaviors. Table II provides a summary and comparison of the control techniques evaluated using the NCD simulator. It shows the time required for 10 and 20 robots to identify and reach the target. Each value is the mean of 30 repetitions of the simulation, with standard deviation in parentheses. The error estimate for these mean values is $\sqrt{30}$ times smaller than the standard deviations listed here. For comparison, if every nanorobot passing through the vessel found the target, 20 nanorobots would arrive at the target in about $0.2 \mathrm{~s}$. As one would expect, enabling nanorobots to detect and follow gradient concentration increases the probability for nanorobots to find the target, where in comparison with random motion the nanorobots show a better performance of $23 \%$. Further, for gradient with "attractant", we see that using the signals allows the nanorobots to find and reach the target in the 3D workspace $46 \%$ faster than that with random motions. This is a remarkable improvement in performance for response time. 


\section{CONCLUSION AND REMARKS}

The development of nanorobots may provide remarkable advances for diagnosis and treatment of cancer. Using chemical sensors they can be programmed to detect different levels of E-cadherin and beta-catenin in primary and metastatic phases. Our work has shown a comprehensive methodology on tracking single tumor cell in a small venule, where nanorobots using communication techniques to increase their collective efficiency. The simulation has clearly demonstrated how better time responses can be achieved for tumor detection, if chemical signals are incorporated as part of nanorobot control strategy. As observed in the study, the follow gradient with attractant signal is a practical method for orientation and coordination of nanorobots. It has enabled a better performance for nanorobots to detect and reach cancerous targets. This approach can be useful in the treatment of many patients for a detailed examination and intervention.

A single tumor cell can be characterized as a typical endothelial cell mutation with profound consequences for patients suffering from cancer. Endothelial cells have a large number of functions and may play an important role in human health. They also serve as part of the structure forming the inside blood vessels, which are spread throughout every single organ or system comprising our body. An abnormal cell mutation and reproduction can represent a wide variety of malignant cases in the oncology field. Thus, a better understanding and the development of new tools based on nanotechnology through chemical sensors may represent important advances to identify, and combat the initial stage of tumor development. Nanorobots can help with significant improvement on cell therapy techniques, and unprecedented positive results to save lives.

\section{ACKNOWLEDGMENTS}

We thank A. Casal, R.A. Freitas Jr., L.C. Kretly, N. Martins, D. Oetomo, L. Rosen, and J. Shi, for helpful comments.

\section{REFERENCES}

[1] S.P. Leary, C.Y. Liu, M.L.I. Apuzzo, "Toward the emergence of nanoneurosurgery: Part III - Nanomedicine: Targeted nanotherapy, nanosurgery, and progress toward the realization of nanoneurosurgery", Neurosurgery, vol. 58, no. 6, pp. 1009-1025 Jun. 2006.

[2] P. Couvreur, C. Vauthier, "Nanotechnology: Intelligent design to treat complex disease", Pharmaceutical Research, vol. 23, no. 7, pp. 14171450 Jul. 2006.

[3] R. A. Freitas Jr., "Nanomedicine”, Vol. I: Basic Capabilities, Landes Bioscience, 1999, "Nanomedicine”, Vol. IIA: Biocompatibility, Landes Bioscience, 2003, http://www.nanomedicine.com.

[4] K. Morris, "Macrodoctor, come meet the nanodoctors", The Lancet, 357:778, 2001.

[5] National Institutes of Health Roadmap: Nanomedicine, 2003, http://nihroadmap.nih.gov/nanomedicine/index.asp.

[6] G.M. Patel, G.C. Patel, R.B. Patel, J.K. Patel, M. Patel, "Nanorobot: A versatile tool in nanomedicine", Journal of Drug Targeting, 14 (2): 63-67 Feb 2006.

[7] J. Howard, "Molecular Motors: Structural Adaptations to Cellular Functions", Nature 389:561-567, 1997.
[8] C. Montemagno, G. Bachand, "Constructing nanomechanical devices powered by biomolecular motors”, Nanotechnology 10:225-231, 1999.

[9] Adriano Cavalcanti, Waren W. Wood, Luiz C. Kretly, Bijan Shirinzadeh, "Computational Nanomechatronics: A Pathway for Control and Manufacturing Nanorobots", IEEE CIMCA Int'l Conf. on Computational Intelligence for Modelling, Control and Automation, IEEE Computer Society, Sydney, Australia, November 2006, www.nanorobotdesign.com.

[10] A. Cavalcanti, R. A. Freitas Jr., "Nanorobotics Control Design: A Collective Behavior Approach for Medicine", IEEE Transactions on NanoBioScience, Vol. 4, no. 2, pp. 133-140, June 2005.

[11] R. Weiss, T. F. Knight Jr., "Engineered Communications for Microbial Robotics", In Proc. of Sixth Intl. Meeting on DNA Based Computers (DNA6), 2000

[12] A. Cavalcanti, "Assembly Automation with Evolutionary Nanorobots and Sensor-Based Control applied to Nanomedicine", IEEE Transactions on Nanotechnology, Vol. 2, no. 2, pp. 82-87, June 2003.

[13] M.J. Zhang, C.L. Sabharwal, W. Tao, T.J. Tarn, N. Xi, G. Li, "Interactive DNA sequence and structure design for DNA nanoapplications", IEEE Transactions on Nanobioscience, vol. 3, no. 4, pp. 286-292 Dec. 2004.

[14] T. Toth-Fejel, "Agents, Assemblers, and ANTS: Scheduling Assembly with Market and Biological Software Mechanisms", Nanotechnology, 11, pp. 133-137, 2000.

[15] W. J. Li, N. Xi, W.K. Fung, T.S. Wong, "Nanorobotics and Nanomanipulation", Encyclopedia of Nanoscience and Nanotechnology, American Scientific Publishers, vol. 7, no. 15, pp. 351-365, 2004.

[16] R.B. Hazana, G.R. Phillipsa, R.F. Qiaoa, L. Nortonb, S.A. Aaronsona, "Exogenous Expression of N-Cadherin in Breast Cancer Cells Induces Cell Migration, Invasion, and Metastasis", The Journal of Cell Biology, Volume 148, Number 4, 779-790, Feb. 2000.

[17] R. Wasielewski, A. Rhein, M. Werner, G.F. Scheumann, H. Dralle, E. Potter, G. Brabant, A. Georgii, "Immunohistochemical detection of Ecadherin in differentiated thyroid carcinomas correlates with clinical outcome", Cancer Research, Vol 57, Issue 12 2501-2507, American Association for Cancer Research, 1997.

[18] A.S.G. Curtis, M. Dalby, N. Gadegaard, "Cell signaling arising from nanotopography: implications for nanomedical devices", Nanomedicine Journal, Future Medicine, vol. 1, no. 1, pp. 67-72, June 2006.

[19] E. Janda, M. Nevolo, K. Lehmann, J. Downward, H. Beug, M. Grieco, "Raf plus TGF beta-dependent EMT is initiated by endocytosis and lysosomal degradation of E-cadherin", Nature, Oncogene, June 2006.

[20] E Sonnenberg, A. Gödecke, B. Walter, F. Bladt, C. Birchmeier, "Transient and locally restricted expression of the ros1 protooncogene during mouse development", EMBO J., 10(12): 3693-3702, 1991 December.

[21] A. Galstyan, T. Hogg, K. Lerman, "Modeling and Mathematical Analysis of Swarms of Microscopic Robots", IEEE Swarm Intelligence Symposium, pp. 201-208, Pasadena CA, USA, June 2005.

[22] S. Vogel, "Life in Moving Fluids", Princeton Univ. Press, 2nd edition, 1994.

[23] G. E. M. Kamiadakis, A. Beskok, "Micro Flows: Fundamentals and Simulation”, Berlin: Springer, 2002.

[24] H. Yamamoto, S. Uemura, Y. Tomoda, S. Fujimoto, T. Hashimoto, K.Okuchi, "Transcardiac Gradient of Soluble Adhesion Molecules Predicts Progression of Coronary Artery Disease", Int'l Journal of Cardiology 84 (2002) 249-257, 2002.

[25] K. E. Drexler, "Nanosystems: Molecular Machinery, Manufacturing, and Computation”, John Wiley \& Sons, 1992.

[26] R.J. Narayan, "Pulsed laser deposition of functionally gradient diamondlike carbon-metal nanocomposites", Diamond and Related Materials, vol. 14, no. 8, pp. 1319-1330 Aug. 2005.

[27] R.J. Narayan, P.N. Kumta, C. Sfeir, D.H. Lee, D. Olton, D. Choi, "Nanostructured ceramics in medical devices: Applications and prospects", JOM, vol. 56, no. 10, pp. 38-43 Oct 2004.

[28] I.D. Villar, I.R. Matias, F.J. Arregui, R.O. Claus, "ESA-based in-fiber nanocavity for hydrogen-peroxide detection", IEEE Transactions on Nanotechnology, vol. 4, no. 2, pp. 187-193 Mar. 2005.

[29] H. C. Berg "Random Walks in Biology", Princeton Univ. Press, 2nd edition, 1993. 